\title{
Chapter 11 \\ Emerging Fields of Colloidal \\ Nanophotonics for Quality Lighting \\ to Versatile Lasing
}

\author{
Hilmi Volkan Demir
}

\begin{abstract}
Solution-processed semiconductor nanocrystals have attracted increasingly greater interest in optoelectronics including color conversion and enrichment in quality lighting and display backlighting. Optical properties of these colloidal nanocrystals can be conveniently controlled by tailoring their shape, composition, and size in an effort to realize high-performance light generation and lasing. We now witness the expanding deployment of semiconductor nanocrystals in consumer products being adapted by giant electronics companies. Based on the rational design and control of excitonic processes in these nanocrystals, it is possible to achieve highly efficient light-emitting diodes and optically pumped lasers. In this chapter, we introduce an emerging field of nanocrystal optoelectronics with applications from quality lighting to versatile lasing. We look into the performance limits of color conversion using colloidal nanocrystals. Here we introduce a new concept of all-colloidal lasers developed by incorporating nanocrystal emitters as the optical gain media intimately into fully colloidal cavities. As an extreme case of solutionprocessed tightly-confined quasi-2D colloids, we also show that the atomically flat nanoplatelets uniquely offer record high optical gain coefficients and ultralow threshold stimulated emission. Given the recent accelerating progress in colloidal nanophotonics, solution-processed quantum materials now hold great promise to challenge their conventional epitaxial counterparts in the near future.
\end{abstract}

Keywords Colloidal nanophotonics · Colloidal quantum dots · Colloidal quantum wells · Semiconductor nanocrystals · Nanoplatelets · All-colloidal lasers

\footnotetext{
H. V. Demir $(\square)$

LUMINOUS! Center of Excellence for Semiconductor Lighting and Displays, NTU - Nanyang Technological University, Singapore, Singapore
}

UNAM - Institute of Materials Science and Nanotechnology, Bilkent University, Ankara, Turkey e-mail: volkan@stanfordalumni.org

B. Di Bartolo et al. (eds.), Quantum Nano-Photonics, NATO Science for Peace 


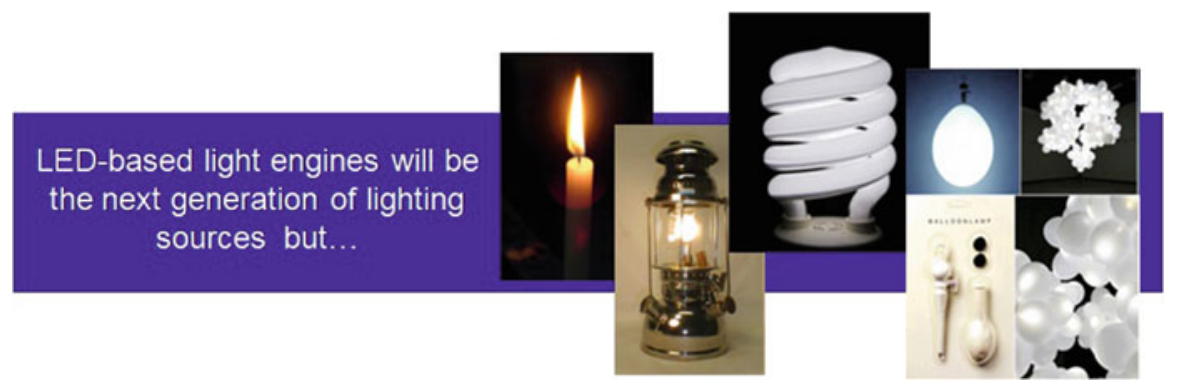

Fig. 11.1 Evolution of light sources: from candles as blackbody radiators to light-emitting semiconductor devices of LEDs as the next-generation light engines

\subsection{Color Conversion and Enrichment with Colloidal Nanocrystals}

\subsubsection{LED Lighting and Photometric Benchmarking}

Artificial lighting uses about one fifth of the global electricity generation worldwide. This consumption is even higher in developing parts of the world, exceeding one third of the electricity generation locally. This can be reduced by over $50 \%$ (and in fact in principle much more depending on the level of improvement in photon management) with the performance levels to be met using semiconductor lightemitting diodes (LEDs) [1]. To this end, the LED-based light engines are targeted to serve as the next-generation lighting sources (Fig. 11.1). However, for a largescale deployment of LEDs, it is not only about the energy efficiency and the optical output power per unit cost; the quality lighting also critically matters. Increasing visual acuity and color perception is hence key to the wide-scale penetration of LEDs into the general lighting market.

For visual acuity, in addition to the efficiency of a light source, the perceived level of efficiency is important. This is related to the sensitivity curves of the human eye, which show the spectrally-varying sensitivity level given the lighting condition (Fig. 11.2). The luminous efficacy of optical radiation (LER) quantifies the perceived optical efficiency (in lumens per optical Watt), while the luminous efficiency (LE) gives the electrical one. LE is basically the perceived optical output power per electrical input power (in lumens per electrical Watt). When there are sufficiently many photons making to a human eye, like on a sunny day, we have the photopic sensitivity curve, which corresponds to the photon-adapted vision, peaking at about $555 \mathrm{~nm}$. As we have fewer and fewer photons collected by the eye, however, the sensitivity curve shifts toward shorter wavelengths, going through the mesopic region. When very few photons are available to the eye, like at a fullmoon night, we lose the color perception but can still see - this is where we have the scotopic curve in effect, peaking at about $505 \mathrm{~nm}$. This corresponds to the darkadapted vision. Between the photopic and scotopic peaks, there is a major shift 
Fig. 11.2 Human eye sensitivity, shifting with the lighting intensity level: photopic, mesopic and scotopic sensitivity curves

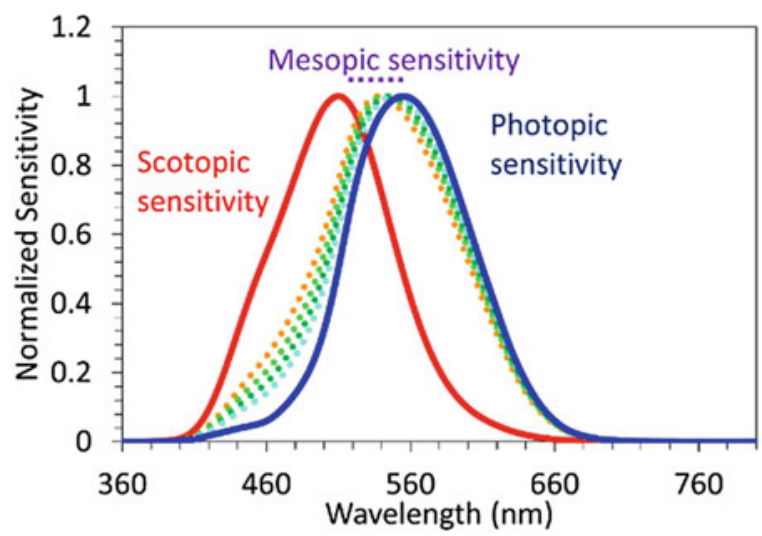

of about $50 \mathrm{~nm}$, which makes a substantial difference for the spectral content of a light source in terms of the perceived efficiency given the amount of photons available. In between these two extremes there is the transition region of mesopic curves. Because the sensitivity curves move with the lighting level, once can achieve spectrally enhanced lighting with careful spectral management of the lighting for the same of level optical power.

In addition to the visual acuity, we have equally important the visual performance, which is related to the shades of white light. This is quantified by the correlated color temperature (CCT). The weaker bluish tint in the white shade, the warmer white light is, corresponding to the lower CCT. This is essential to avoid disrupting biorhythm (circadian cycle) of the human clock (day-night). Finally, as another important aspect of quality lighting, the visual comfort requires good color rendition. This is related to the capability of a light source to reflect the true colors of an illuminated object. There are various metrics that have been defined to represent the level of color rendering including color rendering index (CRI) and color quality scale (CQS). This family of figure-of-merits is closely linked to human emotions triggered by light.

There are two mainstream strategies of generating white light using LEDs. In one approach, commonly known as the multi-chip approach, there are multiple LED chips, typically comprising of red-, green- and blue-emitting LEDs used together to make a white LED platform. Multiple LED chips emitting in different colors age at different rates. To keep the operating color point the same over time, the driving circuitry is typically complicated and there is a cost issue related with such complex drivers. On top of that, there is a green gap problem: green LEDs suffer efficiency, making it challenging to cover the green spectral region.

On the other hand, an easy and convenient approach for white light generation with LEDs is the so-called 'color conversion', which allows for using colorconverting phosphors together with a pump LED emitting at a short wavelength, for example, more commonly in blue or cyan and possibly in near-UV. Typical colorconverting materials contain rare earth elements as the emission centers doped in 


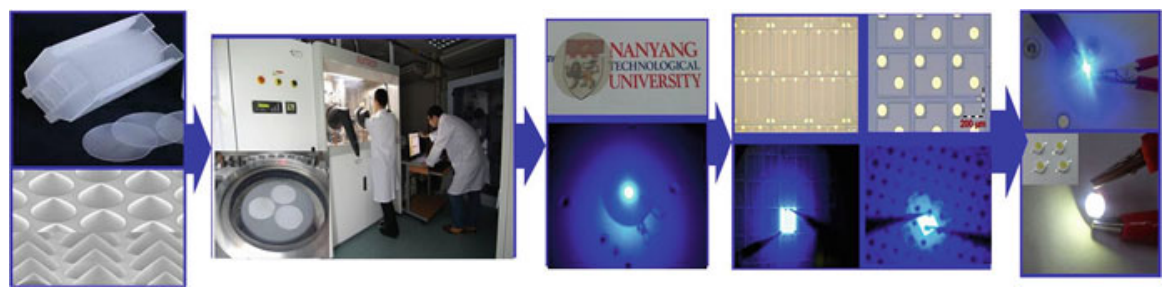

Fig. 11.3 Making a color-conversion white LED from the MOCVD growth to chip fabrication and packaging with the color convertors

a host matrix. This approach is a low-cost alternative to the multichip approach. However, there is a fundamental energy loss associated with conversion from the high-energy photons to the low-energy ones in the process of color conversion. Today the white LEDs most commonly found in the market are the color conversion LEDs. Figure 11.3 shows the processing steps of making a color-conversion LED starting with epitaxial growth of III-nitride epitaxy on sapphire using metalorganic chemical vapor deposition (MOCVD), continuing with the chip fabrication, and finally integrating with the color convertors at the chip level.

In color conversion, typically broad emitters of phosphors are used. They are easy to generate white light. However, it is difficult to control their spectral features precisely. For example, yellow phosphors are often employed, which would typically result in a low color rendering index of $\sim 70$. Using yellow phosphors, one would commonly achieve cool white appearance due to high correlated color temperature in the range of $\sim 4000-8000 \mathrm{~K}$. To address this difficulty, red-green phosphors can be preferred. Surely, this will allow for improved color rendering, exceeding 80 and even possibly reaching 95 in CRI while the CCT can be kept $<3500 \mathrm{~K}$; but, this comes at the penalty of a reduced luminous efficacy level of optical radiation due to the inevitable red tail, typically falling below $300 \mathrm{~lm} / \mathrm{W}_{\text {opt }}$. In addition, there are other problems related to the phosphor host and dopants including the granularity of their powder form and the resulting undesired scattering, the narrow absorption band of their rare earth dopants, and finally the supply security of the rare earth elements, which has become a major problem more recently.

\subsubsection{Paradigm Shift with Narrow Quantum Emitters of Nanocrystals}

Solution-processed semiconductor nanocrystals have been attracting increasingly a greater deal of interest in color conversion and enrichment for quality lighting and display backlighting. Optical properties of these colloidal materials can be conveniently controlled by tailoring their shape, composition, and size [2]. As 
an alternative to broad emitters, narrow emitters of these nanocrystals offer the possibility to obtain photometrically ultimate performance. This is enabled by the fact that it is possible to control precisely the spectrum of such narrow quantum emitters.

Using various combinations of semiconductor quantum dot nanophosphors, it is possible to achieve warm white light with CCT $<3500 \mathrm{~K}$, CRI $>80$, and LER $>300 \mathrm{~lm} / \mathrm{W}_{\text {opt }}$, all at the same time [3]. This is a superior level of photometric performance compared to what can be possibly achieved with rare earth phosphors. Previously, there were concerns about their large scale production. Now this type of difficulties has been addressed. We now witness the expanding deployment of semiconductor nanocrystals in consumer products being adapted by electronics companies.

High optical quality white LEDs using quantum dot based nanophosphors with a carefully designed spectral content of emission has been widely investigated and a detailed review can be found in Demir, H.V., et al. Nano Today 6, 632 (2011) [3]. This is indeed a paradigm shift from continuous broad emitters to discontinuous narrow emitters of the right strategic combination.

With semiconductor nanocrystals, we can now routinely achieve high crystal quality. This is fast and low-cost solution-based processing. Using colloidal synthesis, shape, size and composition control and tuning are possible to target specific excitonic properties. Among the important advantages of semiconductor nanocrystals that are relevant to white light generation are the tunable absorption edge/emission peak, broadband absorption spectrum, narrow emission spectrum, high photoluminescence quantum efficiency, and stability against photobleaching (compared to organic counterparts). With semiconductor nanocrystals, one can reach reasonably high levels of quantum yield exceeding $95 \%$ in solution (using Cdcontaining dots) with adjustable optical spectrum. In addition to being easy to tune, these nanocrystals offer color purity as a result of their spectrally narrow emission. They provide strong absorption, which is broad-band, whereas phosphors possess narrow absorption bands. This means that it is not only the emission band that one needs to get it right for white light generation, but it is also the absorption band that has to be right to match the emission wavelength of the pump LED.

As a result, semiconductor nanocrystal color convertors, with a careful design of the spectral content, enable high-quality white light having high color rendering index (for most natural objects) above 90, warm white shade at a low correlated color temperature below $3500 \mathrm{~K}$, and a high luminous efficacy of optical radiation above $350 \mathrm{~lm} / \mathrm{W}_{\text {opt }}$. They also allow for high scotopic-to-photopic ratio (S/P) above 2.5 for spectral enhanced lighting. Therefore, photometrically, the nanocrystals provide the ability to outperform the phosphors. Furthermore, semiconductor quantum dots materials are abundant, whereas the rare-earth elements used in the conventional phosphors of LEDs are scarce. To date examples of commercialization and prototypes for general lighting and backlighting include the remote phosphor for general lighting and the backlighting unit for liquid crystal displays (LCD), which has been indeed commercially successful. In fact, in addition to white light generation, color enrichment has become also very important in the LCD 
backlighting. Here the color purity (with a full-width half-maximum of $30 \mathrm{~nm}$ ) is the key and using saturated red, green and blue color components is necessary to span a larger color space than that required by the national TV standards.

When integrated with blue LEDs, at least the red- and green-emitting nanocrystals are needed to make reasonable quality white light source. Adding in also the yellow nanocrystals is essential to make it ultimately high quality. In operation, these nanocrystals are optically pumped by the electroluminescence of their integrated LED, which is electrically driven. In this process, the physical mechanisms involved are the optical absorption and emission in the nanocrystals. The photoluminescence from the nanocrystals together with the electroluminescence form the LED collectively generates the white light. In addition, there are the undesired processes including the reabsorption of the photons emitted by the nanocrystals and dipoledipole interaction (Förster-type energy transfer - FRET) between the nanocrystals. The reabsorption basically decreases the optical output power, while this type of FRET process among nanocrystals makes photo-generated excitons to be quickly zipped into nearby nanocrystals. If there are defected subpopulations of neighbor nanocrystals, then these transferred excitons are trapped and can undergo nonradiative recombination process and these defected nanocrystals thus act as exciton sinks that quench emission. In other words, their effective volume to trap excitons widely expands a result of the FRET process. In summary, in the process of while light generation collectively with the nanocrystal, the journey of an exciton in the nanocrystals includes exciton generation, exciton recombination, exciton transfer (FRET), and exciton trapping. Figure 11.4 illustrates the desired photoluminescence from nanocrystals in addition to the adverse energy transfer between them.

One important challenge with using semiconductor nanocrystals is that they are in dispersion. This means that, as they are synthesized, they are naturally available in solvents. This is not compatible with the existing backend process lines in LED packaging, which uses phosphors in the powder form. For that, the phosphors powder is dispersed in host organics, which is typically silicone, and then this mixture is dispensed directly and cured on the top of LED chips. To adopt

Fig. 11.4 Simple illustration of the concept of color-conversion white LEDs integrating the red- and green-emitting nanocrystals, which depicts the optical pumping by a blue LED chip and the resulting red and green photoluminescence from the nanocrystals, along with the undesired energy transfer from a green nanocrystal to a red one

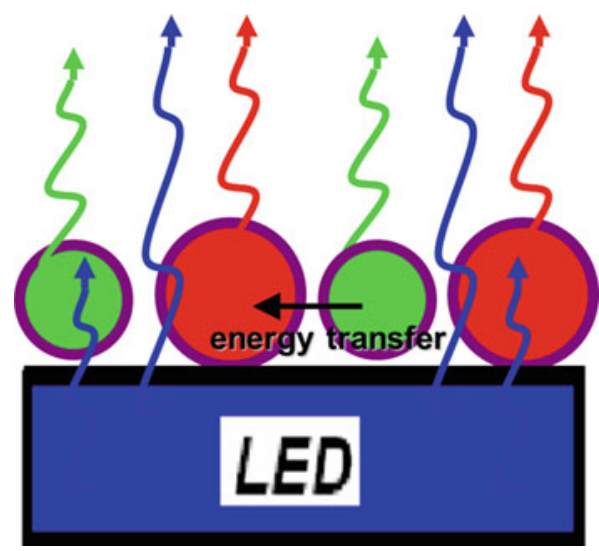


Fig. 11.5 Examples of nanocrystal sheets (monocolor and bi-color)

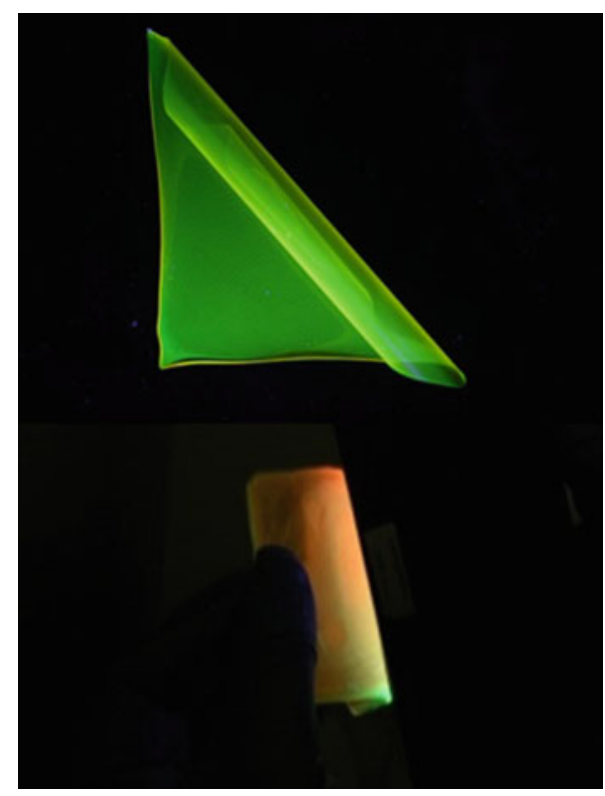

this process, we developed macrocrystals of nanocrystals that allow to embed the semiconductor nanocrystals into the large size crystals wrapping around the tiny nanocrystals and protecting them. These macrocrystals can then be grinded into micro-powder to be mixed with silicone and conveniently dispensed in the available process line.

Another approach is to make standalone nanocrystal films to be used as remote color convertors (Fig. 11.5). This type of nanocrystal sheets can be made quite large in size, in the range of over half a meter by over half a meter, and can be directly used as surface emitters for color conversion in lighting and color enrichment in LCD electronic displays. They can be mono-color or can be made in multiple colors, for example, integrating two layers on top of each other. Figure 11.5 shows proofof-concept demonstrations of a green-emitting standalone nanocrystal sheet along with a bi-color (red and green) version - here one can see the dual color emission from a corner of the bent region.

\subsubsection{Limits of Color Conversion with Nanocrystals}

It is clear that nanocrystals provide us with the ability to carefully engineer the emission spectra to generate white light and optimize the photometric figure of merits, which would otherwise be impossible in the case of broadly-emitting phosphors. This is simply owing to the unique narrow emission of nanocrystals. However, the scientific questions remain: how well can we can do in practice 
Fig. 11.6 Photometrically high-performance color-conversion white LED generated with using nanocrystal emitters

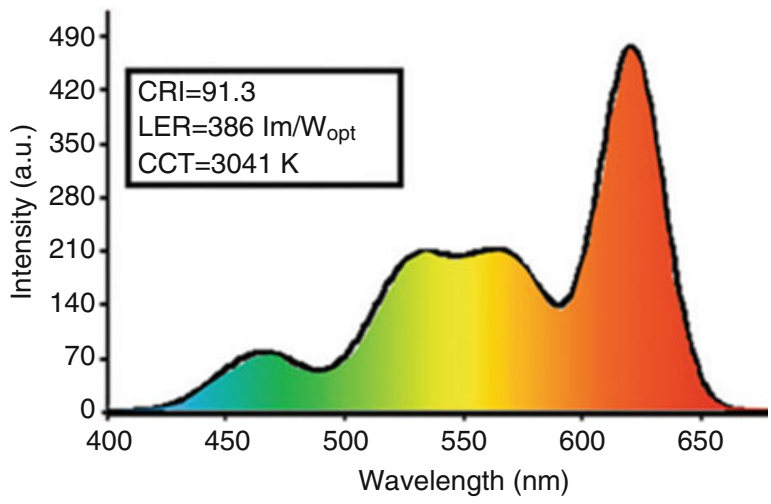

and what are the fundamental limits and trade-offs in terms of the photometric performance when using nanocrystal integrated LEDs? To this end, we investigated what is doable and how, and what is the ultimate performance level that we can realize. To systematically study this problem, we set an ambitious set of photometric targets including CRI $\geq 90, \mathrm{LER} \geq 380 \mathrm{~lm} / \mathrm{W}_{\text {opt }}$, and CCT $<4000 \mathrm{~K}$ [3]. What we found is that surprisingly only a very small fraction (only $0.001 \%$ ) of over 230 million designs reach this performance levels. Obtaining highly efficient white light sources requires spectra that are carefully tuned and precisely controlled.

Extraordinarily high photometric performance is possible using nanocrystals (Fig. 11.6). This first necessitates a strong red component, which needs to be sharp in emission. It turns out that the red component has a critical emission wavelength at $620 \mathrm{~nm}$, which is as far as possible into the red to make it warm white light but not too much to avoid reduction in the spectral efficiency. This color component has to hit the target peak emission with a precision of $1-2 \mathrm{~nm}$, which is reproducibly doable for colloidal synthesis of nanocrystals. Otherwise, the penalty paid in terms of the reduction in photometric performance is the highest compared to all other color components. In contrast to the red, the blue component should be the weakest, and the green and blue can fill in the mid-gap at the middle level. With these, using nanocrystal emitters, it is possible to exceed CRI over 90 and LER over $385 \mathrm{~lm} / \mathrm{W}_{\text {opt }}$ for a quite warm white shade at about $3000 \mathrm{~K}$, as depicted in Fig. 11.6.

To further explore the performance limits and trade-offs, we systematically studied the CRI versus LER for the photopic (photon-adapted) vision (Fig. 11.7). We found that CRI above 90 is feasible within a relatively large window of LER. However, we discovered that this comes at the cost of decreasing LER: the higher LER is, the more CRI is to be traded off. Moreover, this fundamental trade-off becomes steeper with decreasing color temperature (for warmer white shades), as seen in Fig. 11.7. 


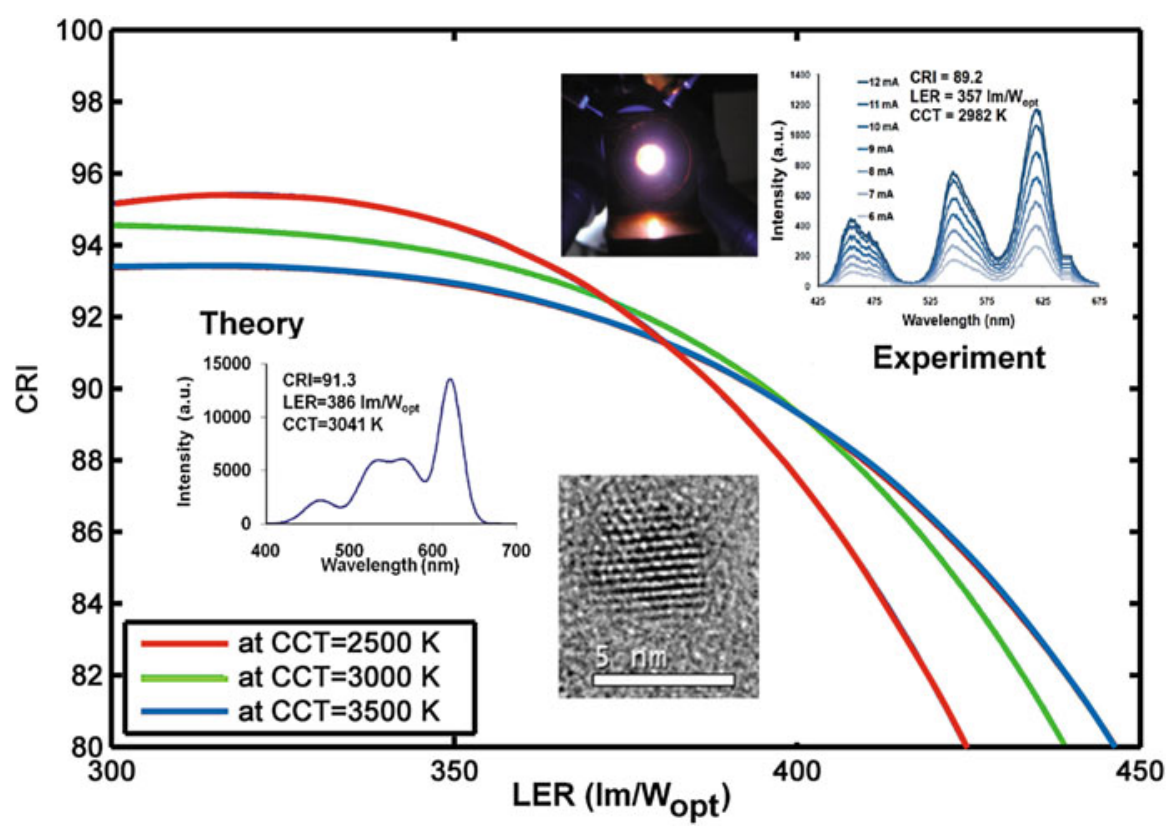

Fig. 11.7 Photometric vision performance limits: CRI vs. LER parametrized with respect to CCT, along with the theoretical and experimental implementations

\subsection{All-Colloidal Lasers of Colloidal Nanocrystals}

\subsubsection{Colloidal Quantum Dots for Lasing}

Based on the rational design and control of excitonic processes in semiconductor nanocrystals, in addition to color-conversion light-emitting diodes, it is possible to achieve highly efficient optically pumped lasers. These provide versatile lasing as a low-cost solution, in principle, essentially on any substrate. In the future, this type of versatile colloidal lasers is considered to find applications for general lighting, among other applications. This is because laser-based lighting is indistinguishable to human eyes compared to other light sources including the LEDs.

For this purpose, we introduce a new concept of all-colloidal lasers developed by incorporating nanocrystal emitters as the optical gain media intimately into fully colloidal cavities [4]. To this end, we use highly efficient $\mathrm{CdSe} / \mathrm{CdS}$ core/shell nanocrystals synthesized with monolayer growth technique. These are polytypic lattice $\mathrm{CdSe} / \mathrm{CdS}$ quantum dots, starting with zinc blende CdSe core and sequentially growing thick $\mathrm{CdS}$ shell in wurtzite. Therefore, the crystal lattice changes from zinc blende to wurtzite. The resulting nanocrystals have highly crystalline structure with a highly monodisperse size distribution (Fig. 11.8). They are almost non-blinking and exhibit near-unity quantum yield. 


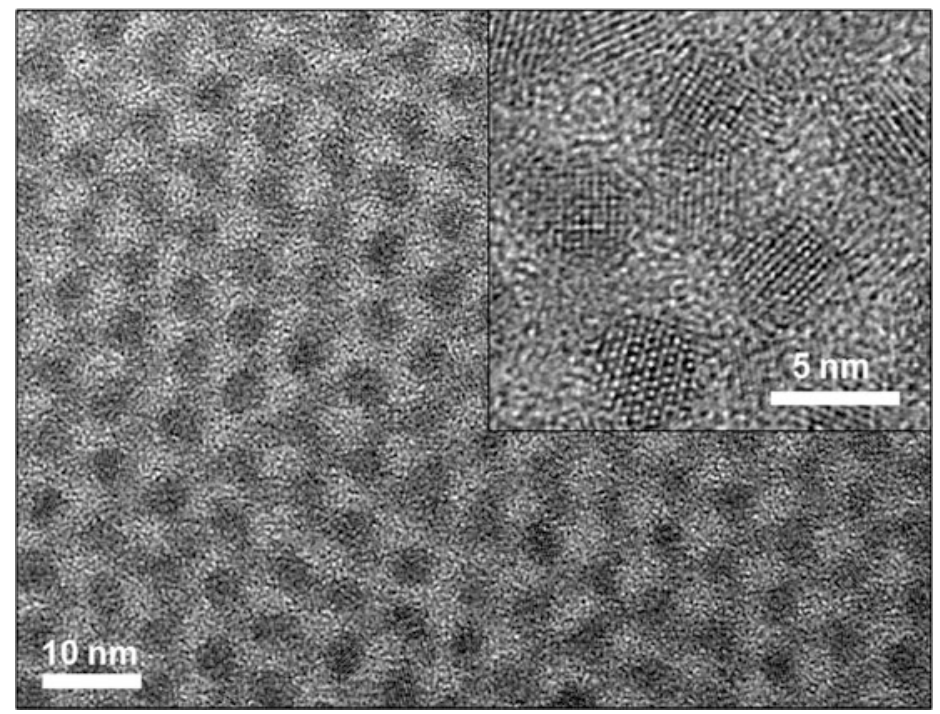

Fig. 11.8 Transmission electron microscopy images of CdSe/CdS core/shell nanocrystals (scale bars: 10 and $5 \mathrm{~nm}$ )

Figure 11.9 illustrates the concept of all-colloidal lasers that integrate quantum dot emitters as the optical gain medium intimately into a fully colloidal cavity. The cavity consists of alternating layers of titania and silica nanoparticles, as can be seen in the cross-sectional scanning electron microscopy image of the colloidal reflectors in the cavity. This laser is made of only solution-processed films including both the colloidal cavity and the colloidal gain. This implies that these lasers can be implemented basically on any platform of any type in principle (flexible, stretchable, etc.) Fig 11.10 shows the emission spectra collected from the all-colloidal laser of quantum dots parameterized with respect to the pump intensity, along with the characteristic spectral narrowing that can be seen in Fig. 11.10a. In Fig. 11.10b the integrated intensity distinctly exhibits the threshold and the stimulated emission region taking over after the threshold. A quality laser beam is observed to come out of this optically pumped all-colloidal laser of quantum dots. To the best of our knowledge, this is the first example of all-colloidal lasers.

Although the lasing performance obtained with this all-colloidal laser of quantum dots is one of the best in its class, there are fundamental barriers that stem from the intrinsic properties of this type of colloidal quantum dots. Among them is the large optical gain thresholds (in the range of several $100 \mathrm{~s} \mu \mathrm{J} / \mathrm{cm}^{2}$ under pulsed excitation), which is due to the small optical gain coefficients $\left(\sim 100 \mathrm{~cm}^{-1}\right)$ possible. The root cause for their limited gain is the ultrashort gain lifetimes (typically shorter than $50 \mathrm{ps}$ ), which is constrained by the dominant Auger recombination and small absorption cross-sections. Therefore, an improved colloidal gain medium is required to overcome these barriers. 


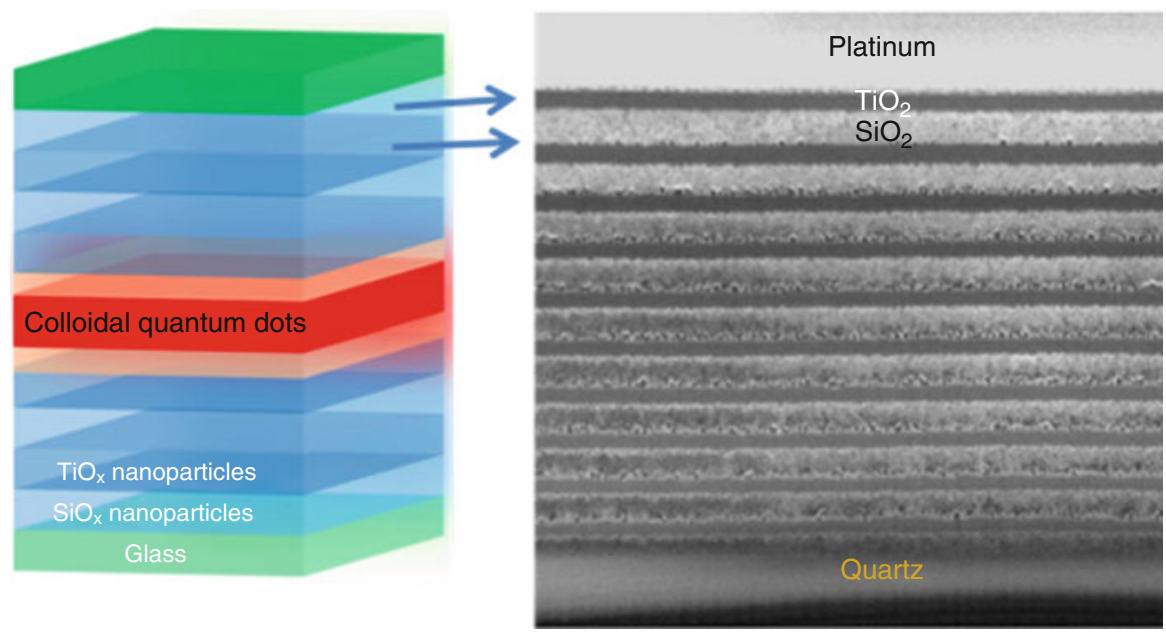

Fig. 11.9 Concept of all-colloidal lasers incorporating quantum dot emitters as the optical gain medium intimately into a fully colloidal cavity made of alternating layers of titania and silica nanoparticles: a simple sketch and a cross-sectional scanning electron microscopy image showing the colloidal reflectors as part of the cavity
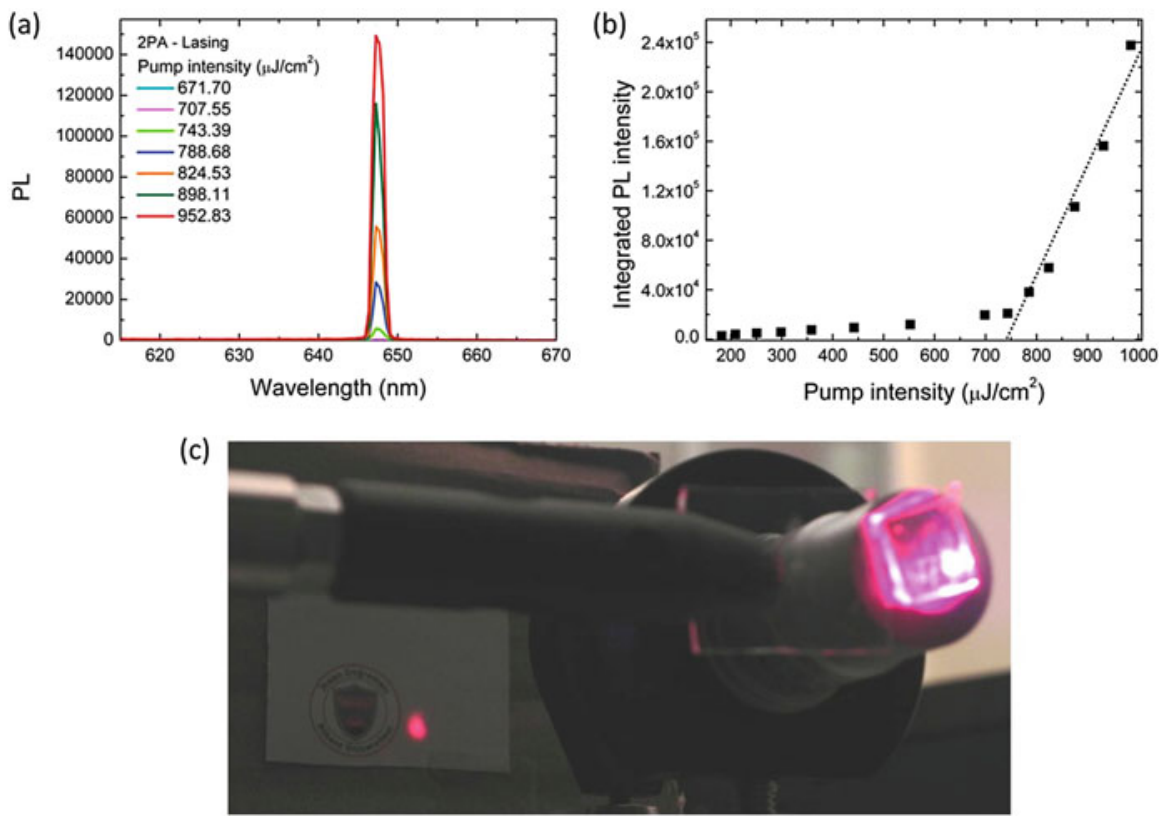

Fig. 11.10 (a) Emission spectra from the all-colloidal laser of quantum dots parameterized with respect to the pump intensity, (b) integrated intensity as a function of the pump intensity, and (c) quality laser beam coming out of the optically pumped all-colloidal laser of quantum dots 


\subsubsection{A New Paradigm Shift with Flat Nanocrystals of Nanoplatelets and Future Outlook}

The need for improved colloidal gain medium brings us to a recent family of semiconductor nanocrystals: colloidal nanoplatelets, which are quasi-two dimensional nanocrystals [5]. These nanoplatelets are basically 'atomically flat'. They do not exhibit size distribution in the vertical thickness; they all have a single "magic" size in the vertical direction. As a result, they exhibit extremely narrow photoluminescence spectrum (with a full-width-at-half-maximum of 7-8 $\mathrm{nm}$ at room temperature). As an extreme case of the solution-processed tightly-confined quasi2D colloids, what we find is that these atomically flat nanoplatelets uniquely offer record high optical gain coefficients and ultralow threshold stimulated emission.

This is indeed a new paradigm shift from colloidal quantum dots to wells possessing flat surfaces and extremely tight confinement. They only suffer homogeneous broadening, enabling their narrow emission. Thanks to their tight confinement, they feature giant oscillator strength and giant absorption cross-section with the lateral area that can be extended over $100 \mathrm{~s}$ of nanometers on each side. These extraordinary properties translate to the reduced optical gain thresholds (in the range of $\sim 10 \mu \mathrm{J} / \mathrm{cm}^{2}$ under pulsed excitation) and large optical gain coefficients $\left(\sim 1000 \mathrm{~cm}^{-1}\right)$, accompanied with long gain lifetimes $(\sim 150 \mathrm{ps})$.

There properties motivate us for all-colloidal laser made of nanoplatelets [6]. Figure 11.11 shows the characteristic emission spectra from such an all-colloidal laser of quantum wells parameterized with respect to the pump intensity and the integrated intensity as a function of the pump intensity. This is one of the first demonstrations of lasing using core/crown nanoplatelets by single photon absorption pumping. For another demonstration, also see another independent report from Talapin et al. [6]. Two photon absorption based pumping is also possible. This has thus led to also the first demonstration of lasing in the nanoplatelets using twophoton absorption pumping. In operation, record high optical gain was observed, which is $4 \times$ larger than the best in quantum dots and $2 \times$ larger than the best in quantum rods. Systematic variable stripe length study revealed that, while in colloidal dots $\sigma_{\text {gain }}=\sim 150 \mathrm{~cm}^{-1}$ and in colloidal rods $\sigma_{\text {gain }}=300-350 \mathrm{~cm}^{-1}$, the colloidal wells achieve $\sigma_{\text {gain }}>1000 \mathrm{~cm}^{-1}$.

The future outlook includes the advanced heterostructures of nanoplatelets, as visioned in Fig. 11.12. This kind of advanced heteronanoplatelets offers a great deal of photo- and thermal-stability, along with further enhanced gain capabilities. With such an accelerating progress, such solution-processed quantum wells now hold great promise to challenge their conventional epitaxial counterparts in the near future. 

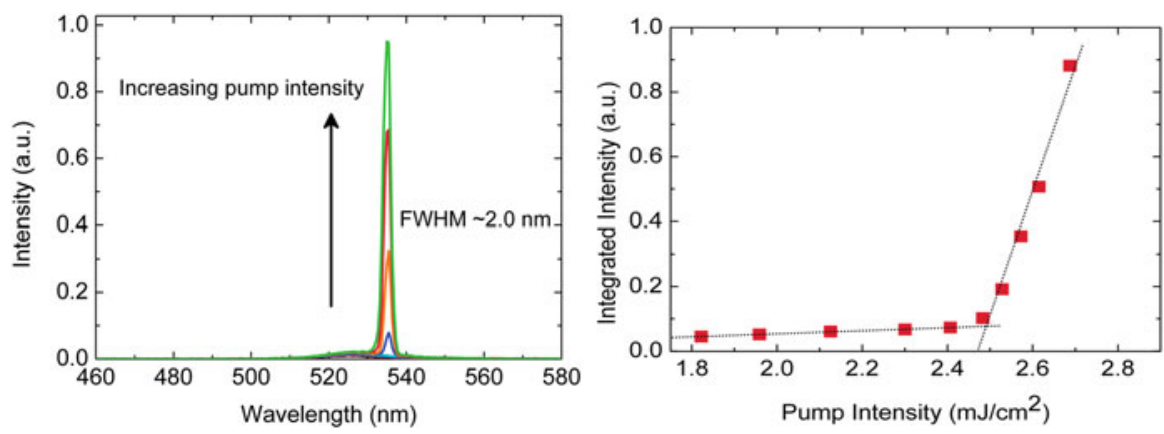

Fig. 11.11 Emission spectra from the all-colloidal laser of quantum wells parameterized with respect to the pump intensity, together with integrated intensity as a function of the pump intensity

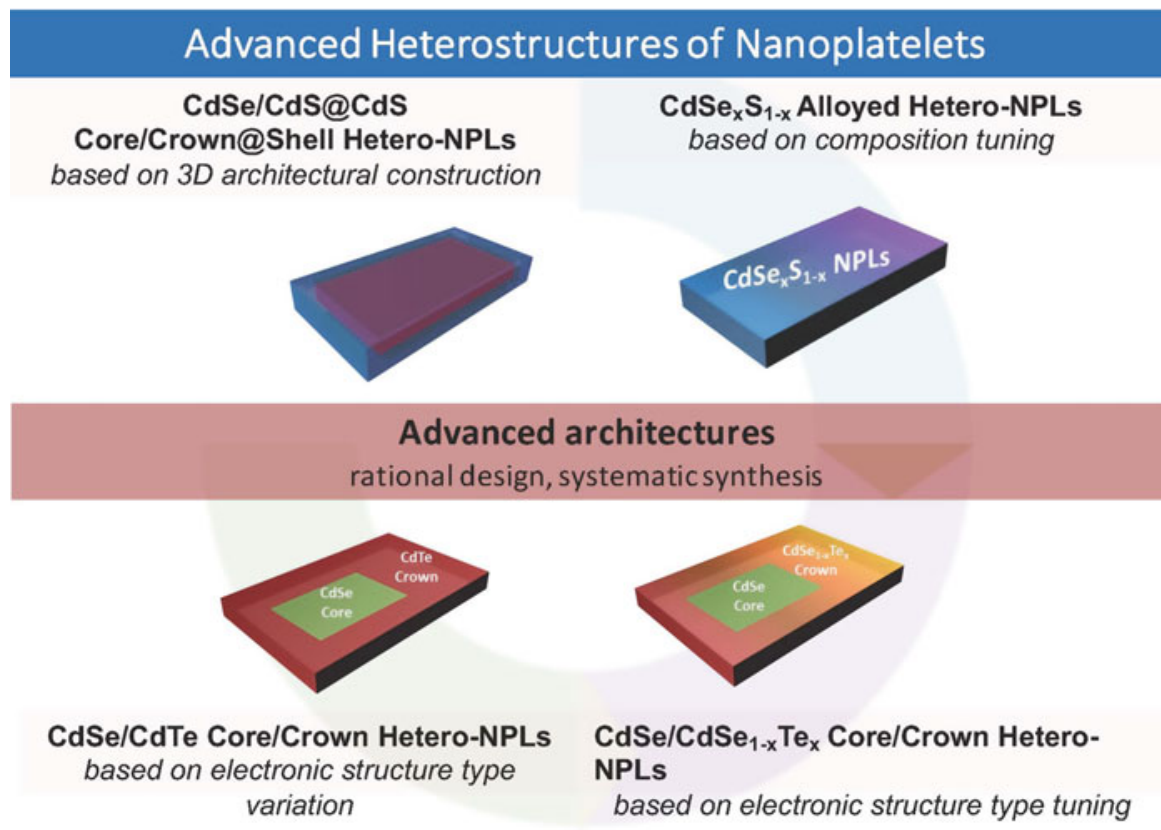

Fig. 11.12 Outlook for advanced heterostructures of nanoplatelets

\section{References}

1. Shubert EF (2006) Light-emitting diodes. Cambridge University Press, Cambridge

2. Gaponenko SV (1998) Optical properties of semiconductor nanocrystals. Cambridge University Press, Cambridge

3. Demir HV et al (2011) Nano Today 6:632; Erdem T, Demir HV (2011) Nat Photonics, 5(3):126

4. Guzelturk B et al (2015) Adv Mater 27:2741

5. Ithurria $S$ et al (2008) J Am Chem Soc 130:16504; and Ithurria $S$ et al (2011) Nat Mater 10:936

6. Guzelturk B et al (2014) ACS Nano 8:6599; also, C. She et al Nano Lett (2014) 14:2772-2777 